\title{
SOME PROBLEMS IN CONFORMAL MAPPING
}

BY

\author{
J. A. JENKINS
}

\section{Chapter I. InTRoduction}

1. The present work is concerned with the conformal mapping of one domain of a certain class into another such domain under given auxiliary conditions. Necessary and sufficient conditions for the possibility of such a mapping will be given in terms of modules of the domains in question. These constitute an extension of the concept of extremal length. The method of extremal length is due to Ahlfors and Beurling [2]. It may be regarded as a development, on the one hand, of the important method initiated independently by Grötzsch [4] and Ahlfors [1] and, on the other, of the work of Beurling [3]. It is further related to some considerations of Teichmüller [5]. (Numbers in brackets refer to the bibliography.)

This introduction will be devoted to an exposition of known properties of extremal length. The remaining three chapters will contain the solution of mapping problems for the pentagon, the hexagon, and the triply-connected domain.

I wish to take this opportunity of expressing my thanks to Professor Ahlfors for the great help and encouragement which he has given me.

2. The general concept of extremal length is introduced as follows: let $\Omega$ be a domain (for convenience we may take it to be bounded) in the $z$-plane containing a family $\Gamma$ of curves $\gamma$. Let $\rho$ be a non-negative function defined in $\Omega$, of integrable square and such that $\int_{\gamma} \rho|d z|$ exists for all $\gamma$ in $\Gamma$ (possibly having the value $+\infty$ ) with $\int_{\gamma} \rho|d z| \geqq 1$. Let g.l.b. $\iint_{\Omega} \rho^{2} d x d y=1 / \lambda$ where $z=x+i y, \rho$ runs through the functions just defined, and $\lambda$ may be $+\infty$. Then $\lambda$ is called the extremal length of the family $\Gamma$.

This quantity $\lambda$ is a conformal invariant. Indeed, let $\Omega$ be mapped conformally on a domain $\Omega^{\prime}$ in the $z^{\prime}$-plane by a function $z^{\prime}=f(z)$, while $z=\phi\left(z^{\prime}\right)$. Let the family $\Gamma$ go into a family $\Gamma^{\prime}$ of curves $\gamma^{\prime}$. Then given a function $\rho(z)$ admissible for $\Omega$ and $\Gamma$, we obtain a function $\rho^{\prime}\left(z^{\prime}\right)=\rho\left(\phi\left(z^{\prime}\right)\right)\left|\phi^{\prime}\left(z^{\prime}\right)\right|$. For this

$$
\int_{\gamma^{\prime}} \rho^{\prime}\left(z^{\prime}\right)\left|d z^{\prime}\right|=\int_{\gamma} \rho(z)|d z|
$$

and $\left(z^{\prime}=x^{\prime}+i y^{\prime}\right)$

$$
\iint_{\Omega^{\prime}}\left(\rho^{\prime}\left(z^{\prime}\right)\right)^{2} d x^{\prime} d y^{\prime}=\iint_{\Omega}(\rho(z))^{2} d x d y .
$$

Presented to the Society, December 30, 1948; received by the editors October 6, 1948. 
In a similar manner to each function $\rho^{\prime}\left(z^{\prime}\right)$ we obtain a function $\rho(z)$. Thus the extremal length is the same in each case.

3. We now proceed to determine certain particular extremal lengths in the case of a quadrangle. By a quadrangle we mean a simply-connected domain with four accessible boundary points or prime ends selected as vertices. Let us suppose the vertices of the quadrangle are numbered 1, 2, 3, 4 in counterclockwise order about the contour.

We shall consider the family $\Gamma$ of curves joining the sides 12 and 34 . These are to be Jordan arcs and are allowed to tend to a prime end at either extremity. Let us call such a curve in the future a $\tilde{J}$-curve. We wish to discuss the corresponding extremal length.

It is well known that we can map a quadrangle $Q$ on a rectangle $R$ in such a way that its vertices $1,2,3,4$ go into the vertices $A_{1}, A_{2}, A_{3}, A_{4}$ of the rectangle. For example, we can first map the quadrangle on a half-plane and then map the latter on a rectangle by using an elliptic function.

Applying a magnification if necessary, we may assume that the side $A_{2} A_{3}$ has length 1 and denote the length of the side $A_{1} A_{2}$ by $l$. Then an extremal metric $\rho$ is provided by $\rho \equiv 1$. Indeed, let us choose a set of Cartesian coordinates with the $x$-axis along $A_{1} A_{2}$ and the $y$-axis along $A_{1} A_{4}$. Clearly for a curve $\gamma$ joining $A_{1} A_{2}$ and $A_{3} A_{4}$,

$$
\int_{\gamma} 1 \cdot|d z| \geqq 1
$$

The corresponding value is

$$
\iint_{R} 1 \cdot d x d y=l
$$

Now let $\rho$ be any other admissible metric and let $\gamma_{x}$ be the intercept made by the rectangle on the ordinate at $x$. Then

$$
\int_{\gamma x} \rho d y \geqq 1 \text {. }
$$

Integrating over the abscissae from 0 to $l$ we obtain

$$
\int_{0}^{l} d x \int_{\gamma_{x}} \rho d y \geqq l
$$

or

$$
\iint_{R} \rho d x d y \geqq l
$$

Now 


$$
\begin{aligned}
0 & \leqq \iint_{R}(\rho-1)^{2} d x d y=\iint_{R} \rho^{2} d x d y-2 \iint_{R} \rho d x d y+\iint_{R} d x d y \\
& \leqq \iint_{R} \rho^{2} d x d y-l
\end{aligned}
$$

Thus $\rho \equiv 1$ does give an extremal metric as stated and any other extremal metric would be almost everywhere equal to 1 . We observe that, in this case, the greatest lower bound is actually a minimum. The extremal length of the above class of curves we may call the extremal distance of the sides 12 and 34 . As we have seen, it is $1 / l$ where $l$ refers to the corresponding rectangle. That a conformally invariant meaning can be attached to $l$ shows, as has long been known, that the ratio of the sides of a rectangle on which a given quadrangle can be mapped is uniquely determined.

4. Now let us suppose we have a second quadrangle $Q^{\prime}$ with vertices $1^{\prime}, 2^{\prime}, 3^{\prime}, 4^{\prime}$ taken in counterclockwise order on the contour as before. Let it be mapped on a rectangle in the $z^{\prime}$-plane having sides $l^{\prime}$ and 1 in place of $l$ and 1. We ask under what conditions the second quadrangle can be mapped conformally into the first in such a way that the side $1^{\prime} 2^{\prime}$ lies along the side 12 and the side $3^{\prime} 4^{\prime}$ lies along the side 34 .

If such a mapping is possible we see that $l \geqq l^{\prime}$. Let the corresponding mapping function be $z=\phi\left(z^{\prime}\right)$. Indeed in the $z$-plane $\rho \equiv 1$ would be an admissible function for the second quadrangle and the corresponding integral

$$
\iint_{Q^{\prime}} \rho^{2} d x d y \leqq l
$$

If we had equality, $l=l^{\prime}$, it is clear that the rectangles would be congruent.

On the other hand, if $l \geqq l^{\prime}$, a mapping as indicated above is obtained simply by laying the second rectangle into the first.

Thus a necessary and sufficient condition for the possibility of such a mapping is that $l \geqq l^{\prime}$. Equality is possible only in the case of conformal equivalence.

5. We have given a relatively complete account of these known results on the quadrangle because they give a broad outline of the methods to be used in the more complicated questions to follow. In the latter not only is there greater technical complication but new phenomena make their appearance, for example, in the question of equality.

\section{Chapter II. The pentagon}

1. In this chapter we consider a mapping problem for a pentagon. Analogously to the quadrangle, a pentagon $\Pi$ is a simply-connected domain with five accessible boundary points or prime ends selected as vertices. These mark off on the boundary the five sides of the pentagon. Let the vertices be $1,2,3,4,5$, taken in counterclockwise order around the boundary. We may 
regard the pentagon as mapped on the upper half-plane so that the vertices lie along the real axis, say at points $z_{1}, z_{2}, z_{3}, z_{4}, z_{5}$ (in order of magnitude).

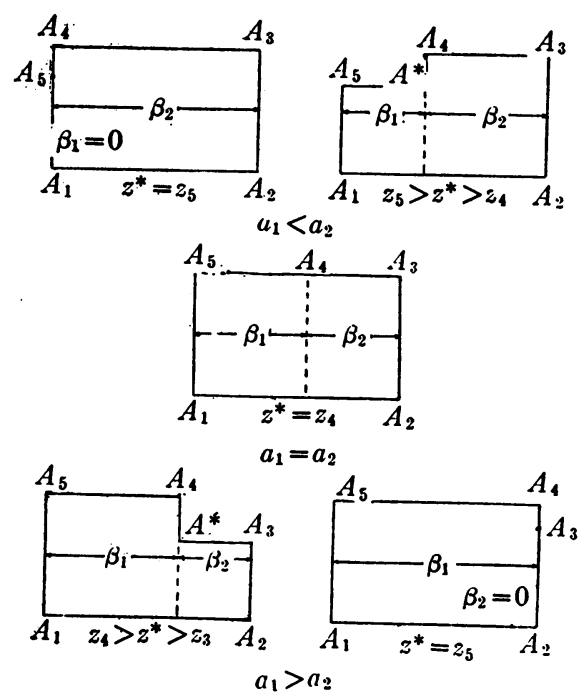

FIG. 1

We shall begin by solving the following extremal problem which we denote by $P\left(a_{1}, a_{2}\right)$.

Problem $P\left(a_{1}, a_{2}\right)$ : Let $C_{1}$ denote the class of $\tilde{J}$-curves $\gamma_{1}$ lying in the pentagon and running from the side 12 to the side 45 . Let $C_{2}$ denote the similar class for the sides 12 and 34 .

Let $\rho$ denote a non-negative function defined over the pentagon, of integrable square and such that the integrals $\int_{\gamma_{1}} \rho|d z|$ and $\int_{\gamma_{2}} \rho|d z|$ exist (possibly having the value $+\infty$ ) with

$$
\int_{\gamma_{1}} \rho|d z| \geqq a_{1}, \quad \int_{\gamma_{2}} \rho|d z| \geqq a_{2}
$$

where $a_{1}, a_{2}$ are certain two non-negative numbers. It is required to determine a $\rho$ such that

$$
\iint_{\mathrm{II}} \rho^{2} d x d y \quad(z=x+i y)
$$

is a minimum. The corresponding minimum will be denoted by $M\left(a_{1}, a_{2}\right)$ and called the module of the pentagon.

The solution of this problem will be given by mapping the pentagon on a suitable canonical domain. This is effective since the problem is immediately seen to be conformally invariant by the same method as in chap. I, §2.

We observe further that the essential quantity is the ratio $a_{1}: a_{2}$. If we have 
the extremal function $\rho$ for a pair $a_{1}, a_{2}$ the corresponding function for the pair $m a_{1}, m a_{2}$ will be $m \rho$.

2. The canonical domain. The canonical domain for the pentagon has one of five possible forms. For $a_{1}<a_{2}$ there are two possibilities. The domain may be a rectangle with corners $A_{1}, A_{2}, A_{3}, A_{4}$ corresponding to 1, 2, 3, 4 and with a point $A_{5}$ on the side $A_{1} A_{4}$ corresponding to $5 . A_{2} A_{3}$ has length $a_{2}$ and $A_{1} A_{5}$ length at least $a_{1}$. Alternatively we may have a hexagon with vertices $A_{1}, A_{2}, A_{3}, A_{4}, A_{5}$ corresponding to $1,2,3,4,5$ at angles of $\pi / 2$ and a sixth vertex $A^{*}$ between $A_{4}$ and $A_{5}$ on the boundary at an angle of $3 \pi / 2$. In this case $A_{1} A_{5}$ and $A_{2} A_{3}$ have respectively lengths $a_{1}$ and $a_{2}$. For $a_{1}=a_{2}$ the domain is a rectangle with corners $A_{1}, A_{2}, A_{3}, A_{5}$ corresponding to $1,2,3,5$ and with a point $A_{4}$ on the side $A_{3} A_{5}$ corresponding to 4 . Both $A_{1} A_{5}$ and $A_{2} A_{3}$ have length $a_{1}=a_{2}$. For $a_{1}>a_{2}$ there are hexagonal and rectangular domains as for $a_{1}<a_{2}$. These possibilities are illustrated in Fig. 1. In any case we denote the perpendicular distances of $A_{4}$ from $A_{1} A_{5}$ and $A_{2} A_{3}$ by $\beta_{1}$ and $\beta_{2}$ respectively.

Before proving that to any pair $a_{1}, a_{2}$ we can obtain a canonical domain, we shall show how the latter provides the solution of the problem $P\left(a_{1}, a_{2}\right)$.

The extremal metric is given in any case by $\rho \equiv 1$. Indeed, let us select a set of Cartesian coordinates with the $x$-axis along $A_{1} A_{2}$ and the $y$-axis along $A_{1} A_{5}$. Clearly

$$
\int_{\gamma_{1}} 1 \cdot|d z| \geqq a_{1}, \quad \int_{\gamma_{2}} 1 \cdot|d z| \geqq a_{2} .
$$

The corresponding value is

$$
\iint_{\pi} 1 \cdot d x d y=a_{1} \beta_{1}+a_{2} \beta_{2}
$$

This is true even for the degenerate cases for if $A_{1} A_{5}>a_{1}$ we have $\beta_{1}=0$ and if $A_{2} A_{3}>a_{2}$ we have $\beta_{2}=0$. To show the minimizing property, let $\rho$ be any other admissible function and let $\gamma_{x}$ denote the intercept made by the domain on the ordinate at $x$. By assumption

$$
\begin{array}{lr}
\int_{\gamma x} \rho d y \geqq a_{1}, & 0<x<\beta_{1}, \\
\int_{\gamma_{x}} \rho d y \geqq a_{2}, & \beta_{1}<x<\beta_{1}+\beta_{2} .
\end{array}
$$

Integrating over these ranges of abscissae we obtain

$$
\begin{array}{r}
\int_{0}^{\beta_{1}} d x \int_{\gamma_{x}} \rho d y \geqq a_{1} \beta_{1}, \\
\int_{\beta_{1}}^{\beta_{1}+\beta_{2}} d x \int_{\gamma_{x}} \rho d y \geqq a_{2} \beta_{2} .
\end{array}
$$


Thus

$$
\iint_{\text {II }} \rho d x d y \geqq a_{1} \beta_{1}+a_{2} \beta_{2}
$$

Now

$$
\begin{aligned}
0 & \leqq \iint_{\Pi}(\rho-1)^{2} d x d y=\iint_{\Pi} \rho^{2} d x d y-2 \iint_{\Pi} \rho d x d y+\iint_{\mathrm{II}} d x d y \\
& \leqq \iint_{\Pi} \rho^{2} d x d y-\left(a_{1} \beta_{1}+a_{2} \beta_{2}\right) .
\end{aligned}
$$

Thus $\rho \equiv 1$ provides an extremal metric and any other extremal metric would be almost everywhere equal to 1 .

We observe that a given pentagon could not be mapped on two distinct canonical domains associated with the same pair $a_{1}, a_{2}$. Indeed, if this were to happen, we would let the domains lie in the $z$ - and $z^{\prime}$-planes, say. They would be related by a conformal transformation $z=\phi\left(z^{\prime}\right)$. Then in the $z^{\prime}$-plane $\rho\left(z^{\prime}\right)=\left|\phi^{\prime}\left(z^{\prime}\right)\right|$ would give an extremal metric, and so be almost everywhere equal to 1 . Being continuous, it would be identically 1 and we should find

$$
\phi\left(z^{\prime}\right)=e^{i \theta} z^{\prime}+c,
$$

$\theta$ real.

Thus the two canonical domains would be congruent.

3. Now we proceed to show that to every ratio $a_{1} / a_{2}$ there exists a canonical domain. Let us regard the mapping of the upper half $z$-plane given by the function

$$
\zeta=C \int_{z_{1}}^{z}\left(\frac{z-z^{*}}{\left(z-z_{1}\right)\left(z-z_{2}\right)\left(z-z_{3}\right)\left(z-z_{4}\right)\left(z-z_{5}\right)}\right)^{1 / 2} d z
$$

where $z^{*}$ is a real value in the interval $z_{3} \leqq z^{*} \leqq z_{5}, C$ is a constant, and we fix the positive determination of the radical between $z_{1}$ and $z_{2}$. By a familiar argument we then see that the upper half-plane is mapped on the types of canonical domains enumerated in $\$ 2$, respectively, according as $z^{*}=z_{5}$, $z_{5}>z^{*}>z_{4}, z^{*}=z_{4}, z_{4}>z^{*}>z_{3}, z^{*}=z_{3}$.

As $z^{*}$ varies from $z_{5}$ to $z_{3}$ the image domain starts out as a rectangle, passes through a series of hexagonal domains and finally becomes a rectangle again. Let the values of the ratio $A_{1} A_{5} / A_{2} A_{3}$ for the extreme rectangles be $k$ and $l(k<l)$. If $a_{1} / a_{2} \leqq k$ the corresponding canonical domain is the rectangle given by $z^{*}=z_{5}$. If $a_{1} / a_{2} \geqq l$ the corresponding canonical domain is the rectangle given by $z^{*}=z_{3}$. As $z^{*}$ varies from $z_{5}$ to $z_{3}$ the ratio $A_{1} A_{5} / A_{2} A_{3}$ varies continuously and so takes all values between $k$ and $l$. It cannot take any value outside this range because the corresponding image domain would not be similar to the rectangle which is the canonical domain there. Moreover the ratio takes every value between $k$ and $l$ once only. Indeed, if we obtained the 
same ratio for two values of $z^{*}$, say $z_{a}^{*}$ and $z_{b}^{*}$, by the uniqueness of the minimizing function the corresponding maps would be one a magnification of the other. We should thus be led to a conformal mapping of the upper half-plane onto itself, leaving invariant the points $z_{1}, z_{2}, z_{3}, z_{4}, z_{5}$ and carrying $z_{a}^{*}$ into $z_{b}^{*}$. This is clearly impossible. Thus to every value of $a_{1} / a_{2}$ with $k \leqq a_{1} / a_{2} \leqq l$ we get a canonical domain of one of the prescribed types. This proves the existence of a solution of the problem $P\left(a_{1}, a_{2}\right)$ in all cases.

4. Now let $\Pi^{\prime}$ be a second pentagon with vertices $1^{\prime}, 2^{\prime}, 3^{\prime}, 4^{\prime}, 5^{\prime}$, the order around the boundary being the same as before. We shall employ for all entities defined above the corresponding notations with primes. We want to investigate when it is possible to map this second pentagon conformally into the first in such a way that the vertex $4^{\prime}$ falls on the vertex 4 and the sides $1^{\prime} 2^{\prime}, 3^{\prime} 4^{\prime}, 4^{\prime} 5^{\prime}$ lie along the sides $12,34,45$.

We state the following result:

THEOREM 1. A necessary and sufficient condition for the possibility of the above mapping is that

$$
M\left(a_{1}, a_{2}\right) \geqq M^{\prime}\left(a_{1}, a_{2}\right)
$$

for all pairs $a_{1}, a_{2}$.

The necessity of this condition is seen at once. Indeed, suppose we have the mapping performed as above. Then any curve of $C_{1}^{\prime}$ is identical with a curve of $C_{1}$ and any curve of $C_{2}^{\prime}$ is identical with a curve of $C_{2}$. Hence any admissible function $\rho$ for $\Pi$ gives an admissible function $\rho^{\prime}$ for $\Pi^{\prime}$ by assigning the same value at the points of $\Pi^{\prime}$. Moreover

$$
\iint_{\Pi} \rho^{2} d x d y \geqq \iint_{\Pi^{\prime}} \rho^{\prime 2} d x d y .
$$

Since there are possibly further admissible functions for $\Pi^{\prime}$ we have

$$
\min _{\rho} \iint_{\Pi} \rho^{2} d x d y \geqq \min _{\rho^{\prime}} \iint_{\Pi^{\prime}} \rho^{\prime 2} d x d y \text {. }
$$

That is,

$$
M\left(a_{1}, a_{2}\right) \geqq M^{\prime}\left(a_{1}, a_{2}\right) .
$$

We now proceed to the proof of the sufficiency of the condition. To every ratio of the interval $k \leqq a_{1} / a_{2} \leqq l(k>0, l<+\infty)$ we select a definite pair $a_{1}, a_{2}$, say the pair for which $a_{1}$ has a given constant value. To this pair will correspond a canonical domain for $\Pi$ and so a pair of numbers $\beta_{1}, \beta_{2}$. Now

$$
\beta_{1}=\left|\Re C \int_{z^{*}}^{z_{5}}\left(\frac{z-z^{*}}{\left(z-z_{1}\right)\left(z-z_{2}\right)\left(z-z_{3}\right)\left(z-z_{4}\right)\left(z-z_{5}\right)}\right)^{1 / 2} d z\right|,
$$




$$
\beta_{2}=\left|\Re C \int_{z_{3}}^{z^{*}}\left(-\frac{z-z^{*}}{\left(z-z_{1}\right)\left(z-z_{2}\right)\left(z-z_{3}\right)\left(z-z_{4}\right)\left(z-z_{5}\right)}\right)^{1 / 2} d z\right| .
$$

Since $z^{*}$ varies continously with $a_{1} / a_{2}$, these are continuous functions of $a_{1} / a_{2}$. Similarly we get a pair of functions $\beta_{1}^{\prime}, \beta_{2}^{\prime}$ depending continuously on $a_{1} / a_{2}$.
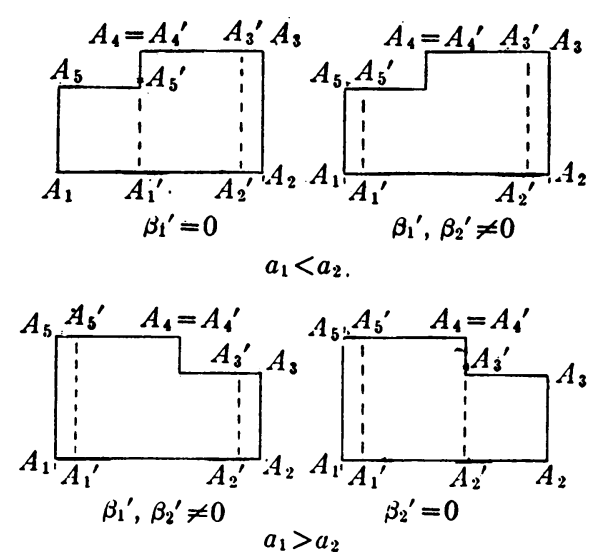

FIG. 2

The condition

$$
M\left(a_{1}, a_{2}\right) \geqq M^{\prime}\left(a_{1}, a_{2}\right)
$$

can be expressed as

$$
a_{1} \beta_{1}+a_{2} \beta_{2} \geqq a_{1} \beta_{1}^{\prime}+a_{2} \beta_{2}^{\prime}
$$

or

$$
a_{1}\left(\beta_{1}-\beta_{1}^{\prime}\right)+a_{2}\left(\beta_{2}-\beta_{2}^{\prime}\right) \geqq 0 .
$$

This can be interpreted as saying that, taken in a Cartesian coordinate system, with axes $O x, O y$, say, the vectors $\left(a_{1}, a_{2}\right)$ and $\left(\beta_{1}-\beta_{1}^{\prime}, \beta_{2}-\beta_{2}^{\prime}\right)$, provided the latter is not the null vector, form an angle not greater than a right angle. Since the first vector must lie in the quadrant $(x+, y+)$ the second cannot lie in the interior of the quadrant $(x-, y-)$. As the ratio $a_{1} / a_{2}$ varies, the end point of the vector $\left(\beta_{1}-\beta_{1}^{\prime}, \beta_{2}-\beta_{2}^{\prime}\right)$ describes a continuous curve. Let us regard under this the image of the segment $k \leqq a_{1} / a_{2} \leqq l$. For this segment the sides $A_{5} A_{1}$ and $A_{2} A_{3}$ of the canonical domain actually have the lengths $a_{1}$ and $a_{2}$. The first end point of the image must fall in the closed quadrant $(x-, y+)$, the second end point in the closed quadrant $(x+, y-)$, since for the first $\beta_{1}=0$ and for the second $\beta_{2}=0$.

If the image curve passes through the origin we have for that value of $a_{1} / a_{2}, \beta_{1}=\beta_{1}^{\prime}$ and $\beta_{2}=\beta_{2}^{\prime}$. If these $\beta_{1}, \beta_{2}$ are greater than zero the two canonical domains will be identical. If $\beta_{1}=0$ then also $\beta_{1}^{\prime}=0$ and the side $A_{5}^{\prime} A_{1}^{\prime}$ is at least $a_{1}$ in length. A similar remark holds if $\beta_{2}=0$. Hence in any case we can 
lay the canonical domain for $\Pi^{\prime}$ into the canonical domain for $\Pi$. To this corresponds the required mapping of $\Pi^{\prime}$ into $\Pi$.

If the image curve does not pass through the origin we can project it continuously from that point onto a circle with center there. This new image will have points in each of the closed quadrants $(x-, y+)$ and $(x+, y-)$. As the continuous image of a connected set it must be connected and it can contain no points in the interior of the quadrant $(x-, y-)$. Thus we conclude that it must contain the whole arc of the circle in the quadrant $(x+, y+)$. Hence we must have vectors $\left(\beta_{1}-\beta_{1}^{\prime}, \beta_{2}-\beta_{2}^{\prime}\right)$ lying in the interior of this quadrant and thus pairs with $\beta_{1}>\beta_{1}^{\prime}, \beta_{2}>\beta_{2}^{\prime}$. Then we can lay the canonical domain for $\Pi^{\prime}$ into the canonical domain for $\Pi$. This is illustrated in Fig. 2. To this corresponds the required mapping of $\Pi^{\prime}$ into $\Pi$. This completes the proof of Theorem 1.

5. We observe further that if we have always the strict inequality

$$
M\left(a_{1}, a_{2}\right)>M^{\prime}\left(a_{1}, a_{2}\right)
$$

we can have only the second possibility above. Thus the interior of $\Pi^{\prime}$ is mapped onto a proper subdomain of the interior of $\Pi$. Conversely this last situation implies that the strict inequality always holds. Indeed, suppose it were possible to map the interior of $\Pi^{\prime}$ onto a proper subdomain of the interior of $\Pi$ under the given conditions on the boundary, while for some pair $a_{1}, a_{2}$

$$
M\left(a_{1}, a_{2}\right)=M^{\prime}\left(a_{1}, a_{2}\right) .
$$

Let the corresponding canonical domains for $\Pi$ and $\Pi^{\prime}$ lie in the $z$ - and $z^{\prime}$-planes respectively. Let the mapping of the canonical domain for $\Pi^{\prime}$ into that for $\Pi$ be given by $z=\phi\left(z^{\prime}\right)$. Then $\left|\phi^{\prime}\left(z^{\prime}\right)\right|$ would be an admissible function for $P^{\prime}\left(a_{1}, a_{2}\right)$ in the $z^{\prime}$-plane. Now

$$
\iint_{\Pi^{\prime}}\left|\phi^{\prime}\left(z^{\prime}\right)\right|^{2} d x^{\prime} d y^{\prime}=\iint_{\Pi^{\prime}} 1 \cdot d x d y \leqq \iint_{\Pi} 1 \cdot d x d y=M\left(a_{1}, a_{2}\right)=M^{\prime}\left(a_{1}, a_{2}\right) .
$$

This means $\left|\phi^{\prime}\left(z^{\prime}\right)\right|$ would be an extremal metric, hence identically equal to 1 (since continuous). Therefore the mapping in question would be a congruent transformation. Equality above would be possible only if the interiors of the canonical domains coincided under this transformation. Thus a mapping of the interior of $\Pi^{\prime}$ onto a proper subdomain of the interior of $\Pi$ would be impossible.

We see that for canonical domains of hexagonal type the above situation implies conformal equivalence of the pentagons. However for rectangular domains we can have equality without conformal equivalence.

We can now state the following complement to Theorem 1.

TheOREм 1a. $\Pi$ and $\Pi^{\prime}$ are conformally equivalent if and only if 


$$
M\left(a_{1}, a_{2}\right)=M^{\prime}\left(a_{1}, a_{2}\right)
$$

for all pairs $a_{1}, a_{2}$.

$\Pi^{\prime}$ can be mapped into $\Pi$ in the required manner with its interior going into a proper subdomain of the interior of $I I$ if and only if

$$
M\left(a_{1}, a_{2}\right)>M^{\prime}\left(a_{1}, a_{2}\right)
$$

for all pairs $a_{1}, a_{2}$.

\section{Chapter III. The hexagon}

1. In this chapter we shall treat a mapping problem for a hexagon which, in a certain sense, is a natural generalization of the problem just treated for the case of a pentagon. Nevertheless in addition to the greater technical detail certain new features are presented which require somewhat more careful treatment.

As we should by now expect, a hexagon $H$ is a simply-connected domain with six accessible boundary points or prime ends selected as vertices. Let the vertices be $1,2,3,4,5,6$, taken in counterclockwise order around the boundary of the domain. These points mark off on the boundary the six sides of the hexagon. We may regard the latter as mapped on an upper half-plane so that the vertices lie at points of the real axis, say $z_{1}, z_{2}, z_{3}, z_{4}, z_{5}, z_{6}$ (in order of magnitude).

We shall regard for the hexagon the following extremal problem which we denote by $P\left(a_{1}, a_{2}, a_{3}\right)$.

Problem $P\left(a_{1}, a_{2}, a_{3}\right)$ : Let $C_{1}$ denote the class of $\tilde{J}$-curves $\gamma_{1}$ lying in the hexagon and running from the side 12 to the side 34 . Let $C_{2}$ denote the similar class for the sides 34 and 56 and $C_{3}$ the similar class for the sides 56 and 12.

Let $\rho$ denote a non-negative function defined over the hexagon, of integrable square and such that $\int_{\gamma_{1}} \rho|d z|, \int_{\gamma_{2}} \rho|d z|, \int_{\gamma_{3}} \rho|d z|$ exist (possibly having the value $+\infty)$. Further we require

$$
\int_{\gamma_{1}} \rho|d z| \geqq a_{1}, \quad \int_{\gamma_{2}} \rho|d z| \geqq a_{2}, \quad \int_{\gamma_{3}} \rho|d z| \geqq a_{3}
$$

where $a_{1}, a_{2}, a_{3}$ are certain three non-negative numbers. We desire to determine a function $\rho$ such that

$$
\iint_{H} \rho^{2} d x d y \quad(z=x+i y)
$$

is a minimum. The corresponding minimum will be denoted by $M\left(a_{1}, a_{2}, a_{3}\right)$ and called the module of the hexagon.

We note that the problem is essentially fixed by the double ratio $a_{1}: a_{2}: a_{3}$. Indeed if $\rho$ is a function solving $P\left(a_{1}, a_{2}, a_{3}\right), m \rho$ will be a function solving 
$P\left(m a_{1}, m a_{2}, m a_{3}\right)$. As before, the solution is given by mapping the hexagon on a suitable canonical domain. Once again this method is applicable because of the conformal invariance of the problem.

2. We now proceed with a discussion of the various possible types of canonical domains. We shall then discuss the functions which give rise to them. Finally we shall show that for any problem $P\left(a_{1}, a_{2}, a_{3}\right)$ we can exhibit a canonical domain leading to the solution.

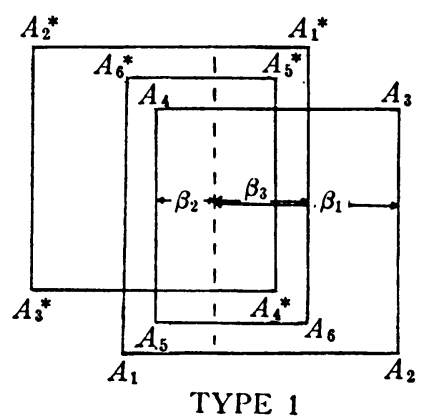

TYPE 1

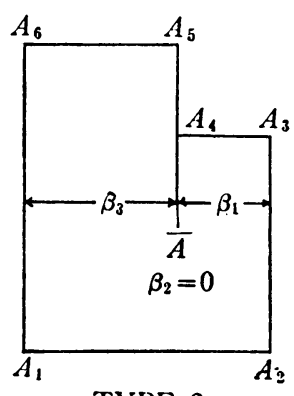

TYPE 2
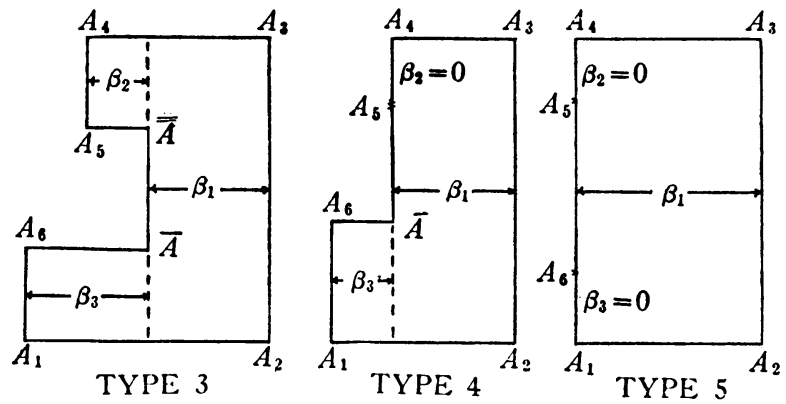

FIG. 3

Any canonical domain belongs to one of five general types. These are illustrated in Fig. 3. The first is the image on a three-sheeted Riemann surface of a two-sheeted covering of the hexagon, the former having a branch point of order three corresponding to a branch point of order two of the latter. There are thus two vertices of this domain corresponding to each one of the hexagon; they are symmetrically placed with respect to the branch point and the angle at each of them is $\pi / 2$. The sides of the canonical domain have the following lengths: $A_{2} A_{3}=A_{2}^{*} A_{3}^{*}=a_{1}, A_{4} A_{5}=A_{4}^{*} A_{5}^{*}=a_{2}, A_{6} A_{1}^{*}=A_{6}^{*} A_{1}=a_{3}$. Moreover these lengths satisfy the inequalities

$$
A_{2} A_{3}+A_{4} A_{5}>A_{6} A_{1}^{*}, \quad A_{4} A_{5}+A_{6} A_{1}^{*}>A_{2} A_{3}, \quad A_{6} A_{1}^{*}+A_{2} A_{3}>A_{4} A_{5} .
$$

The second type of domain has vertices with angles of $\pi / 2$ corresponding to 
the vertices of the hexagon and an extra vertex $\bar{A}$ with an angle of $2 \pi$ in the side $A_{4} A_{5}$. Similar domains would be obtained by having $\bar{A}$ in the sides $A_{2} A_{3}$ or $A_{6} A_{1}$. The sides have lengths $A_{2} A_{3}=a_{1}, A_{4} A_{5}\left(=A_{4} \bar{A}+\bar{A} A_{5}\right) \geqq a_{2}$, $A_{6} A_{1}=a_{3}$. These lengths again satisfy the inequalities

$$
A_{2} A_{3}+A_{4} A_{5} \geqq A_{6} A_{1}, \quad A_{4} A_{5}+A_{6} A_{1} \geqq A_{2} A_{3}, \quad A_{6} A_{1}+A_{2} A_{3}>A_{4} A_{5} .
$$

The third type of domain has vertices with angles of $\pi / 2$ corresponding to the vertices of the hexagon and two extra vertices $\bar{A}$ and $\bar{A}$ with angles of $3 \pi / 2$ in the side $A_{5} A_{6}$. Similar domains would be obtained by having these vertices in the sides $A_{3} A_{4}$ or $A_{1} A_{2}$. In this case the sides have lengths $A_{2} A_{3}=a_{1}$, $A_{4} A_{5}=a_{2}, A_{6} A_{1}=a_{3}$ and these lengths satisfy the inequality

$$
A_{2} A_{3} \geqq A_{4} A_{5}+A_{6} A_{1} \text {. }
$$

The fourth type of domain has vertices $A_{1}, A_{2}, A_{3}, A_{4}, A_{6}$ with angles of $\pi / 2$ corresponding to the vertices $1,2,3,4,6$ of the hexagon, a vertex $\bar{A}$ with an angle of $3 \pi / 2$ between $A_{4}$ and $A_{6}$ and a point $A_{5}$ corresponding to 5 between $A_{4}$ and $\bar{A}$. This may be thought of as being obtained from the preceding case by letting $\bar{A}$ coincide with $A_{5}$. A great variety of similar domains could be obtained, say by letting $\bar{A}$ coincide with $A_{6}$ or starting from one of the alternative cases of the third type. In the case depicted in the diagram the sides have lengths $A_{2} A_{3}=a_{1}, A_{4} A_{5} \geqq a_{2}, A_{6} A_{1}=a_{3}$. These satisfy the inequality

$$
A_{2} A_{3} \geqq A_{4} A_{5}+A_{6} A_{1} \text {. }
$$

The last type of domain is a rectangle with corners $A_{1}, A_{2}, A_{3}, A_{4}$ corresponding to the vertices $1,2,3,4$ of the hexagon. To 5 and 6 correspond points $A_{5}$ and $A_{6}$ on the side $A_{1} A_{4}$. Similar domains would be obtained by letting 1 and 2 or 3 and 4 take the place of 5 and 6 here. Here the sides have lengths $A_{2} A_{3}=a_{1}, A_{4} A_{5} \geqq a_{2}, A_{5} A_{6} \geqq a_{3}$. These satisfy the inequality

$$
A_{2} A_{3}>A_{4} A_{5}+A_{6} A_{1} \text {. }
$$

In the first case we shall denote the perpendicular distances from the point of symmetry to $A_{2} A_{3}, A_{4} A_{5}$, and $A_{6} A_{1}^{*}$ by $\beta_{1}, \beta_{2}$, and $\beta_{3}$ respectively. In the other cases we denote by these symbols the lengths indicated in Fig. 3. The justification for this definition will be seen later.

In each case the extremal metric is given by $\rho \equiv 1$. For example, let us regard a canonical domain of the first type. From the symmetry about the branch point we see that on transforming back to the hexagon this will provide a single-valued function there. By a line through the branch point parallel to the side $A_{2} A_{3}$ (so also to the side $A_{4} A_{5}$, and so on) we can divide the domain into six rectangles, congruent in pairs. The same method as was employed in the case of the pentagon now shows that the required minimum is given by $2\left(a_{1} \beta_{1}+a_{2} \beta_{2}+a_{3} \beta_{3}\right)$. Since the canonical domain is an image of a double covering of the hexagon the corresponding module is $a_{1} \beta_{1}+a_{2} \beta_{2}+a_{3} \beta_{3}$. 
An analogous argument proves that the same expression gives the module for the other types of canonical domains. Further, any other extremal metric would be almost everywhere equal to 1 . Thus, as for the pentagon, two canonical domains for the same hexagon associated with a given double ratio $a_{1}: a_{2}: a_{3}$ would have to be congruent.

3. Our next step is to show that to every double ratio $a_{1}: a_{2}: a_{3}$ there exists a canonical domain.

Let us regard in the first instance the mapping of a two-sheeted covering surface of the upper half-plane with a branch point of second order at $z=z^{*}\left(I z^{*}>0\right)$ by the function

$$
\zeta=C \int_{z^{*}}^{z}\left(\frac{\left(z-z^{*}\right)\left(z-\bar{z}^{*}\right)}{\left(z-z_{1}\right)\left(z-z_{2}\right)\left(z-z_{3}\right)\left(z-z_{4}\right)\left(z-z_{5}\right)\left(z-z_{6}\right)}\right)^{1 / 2} d z
$$

where $C$ is a constant.

The transformation $Z^{2}=z-z^{*}$ will give a simply-covered domain $D$ in the $Z$-plane on which $\zeta$ is regular and single-valued. About $z=z^{*}$ we have the expansion

$$
\begin{aligned}
& C\left(\frac{\left(z-z^{*}\right)\left(z-\bar{z}^{*}\right)}{\left(z-z_{1}\right)\left(z-z_{2}\right)\left(z-z_{3}\right)\left(z-z_{4}\right)\left(z-z_{5}\right)\left(z-z_{6}\right)}\right)^{1 / 2} \\
& \quad=A\left(z-z^{*}\right)^{1 / 2}+\text { higher powers }(A \neq 0) .
\end{aligned}
$$

Thus $\zeta$ has the expansion

$$
\zeta=B\left(z-z^{*}\right)^{3 / 2}+\text { higher powers }
$$

or

$$
\zeta=B Z^{3}+\text { higher powers. }
$$

As $Z$ describes the boundary of $D, z$ describes the real axis twice and a consideration of the behavior of the integrand in the expression for $\zeta$ shows that $\zeta$ describes a curve such as appears as the boundary of a canonical domain of the first type. This curve winds about $\zeta=0$ three times.

Hence $\zeta$ has a triple zero at $Z=0$ and no other zeros in $D$. Thus any chosen determination $\Xi$ of $\zeta^{1 / 3}$ will be a single-valued regular function of $Z$ in $D$. As $Z$ describes the boundary of $D$, $\Xi$ will describe a simple closed curve in the $\Xi$-plane and hence the corresponding interiors will be in $(1,1)$ conformal correspondence. Finally the Riemann surfaces over the $z$ - and $\zeta$-planes will be in $(1,1)$ correspondence, conformal apart from their branch points.

Secondly let us regard the mapping of the upper half $z$-plane given by the function

$$
\zeta=C \int_{z_{1}}^{z}\left(\frac{\left(z-z^{*}\right)^{2}}{\left(z-z_{1}\right)\left(z-z_{2}\right)\left(z-z_{3}\right)\left(z-z_{4}\right)\left(z-z_{5}\right)\left(z-z_{6}\right)}\right)^{1 / 2} d z .
$$


Here $C$ is a constant, $z_{4}<z^{*}<z_{5}$, and we may take the positive determination of the radical on the real axis between $z_{1}$ and $z_{2}$. A consideration of the argument of the integrand shows that the real axis is mapped into a closed contour such as appears as the boundary of a canonical domain of the second type. This function thus gives a mapping on this type of domain. The point $z^{*}$ corresponds to the point $\bar{A}$. Similar image domains would be obtained by taking $z^{*}$ between $z_{2}$ and $z_{3}$ or between $z_{6}$ and $z_{1}$.

Thirdly let us regard the mapping of the upper half $z$-plane given by

$$
\zeta=C \int_{z_{1}}^{z}\left(\frac{\left(z-z^{*}\right)\left(z-z^{* *}\right)}{\left(z-z_{1}\right)\left(z-z_{2}\right)\left(z-z_{3}\right)\left(z-z_{4}\right)\left(z-z_{5}\right)\left(z-z_{6}\right)}\right)^{1 / 2} d z .
$$

Here $C$ is a constant, $z_{5} \leqq z^{* *} \leqq z^{*} \leqq z_{6}$, and we may take the positive determination of the radical on the real axis between $z_{1}$ and $z_{2}$. An argument similar to that above shows that this function in general maps the upper halfplane on a canonical domain of the third type with $z^{* *}$ corresponding to $\vec{A}$ and $z^{*}$ to $\bar{A}$. If $z^{* *}=z_{5}$ we get a canonical domain of the fourth type and if $z^{* *}=z_{5}$ and $z^{*}=z_{6}$ a canonical domain of the fifth type. Naturally we may have $z_{5}<z^{*}=z^{* *}<z_{6}$ in which case the side $\bar{A} \bar{A}$ reduces to a point. $z^{*}$ and $z^{* *}$ may further both coincide with $z_{5}$ or $z_{6}$. Further domains of these types could be obtained by taking $z_{1} \leqq z^{* *} \leqq z^{*} \leqq z_{2}$ and $z_{3} \leqq z^{* *} \leqq z^{*} \leqq z_{4}$.

Now to every point of the upper half-plane including the real axis we have obtained a canonical domain corresponding at least to the ratio $a_{1}: a_{2}: a_{3}$ $=A_{2} A_{3}: A_{4} A_{5}: A_{6} A_{1}$. Also to every pair of points both in one of the segments $z_{1} z_{2}, z_{3} z_{4}$, or $z_{5} z_{6}$ we obtain a canonical domain corresponding to this same ratio.

Let us regard the $\left(z^{* *}, z^{*}\right)$ plane. The pairs of points mentioned above are in $(1,1)$ correspondence in the natural way with the points of the three triangular regions defined by $z_{1} \leqq z^{* *} \leqq z^{*} \leqq z_{2} ; z_{3} \leqq z^{* *} \leqq z^{*} \leqq z_{4} ; z_{5} \leqq z^{* *} \leqq z^{*} \leqq z_{6}$. After a suitable linear reduction we shall join each of these to the upper half $z$-plane so as to lie below the real axis and have the points of its hypotenuse attached to the corresponding intervals of the real axis. Let us denote these triangles by $T_{1}, T_{2}, T_{3}$, their lower vertices by $a, b, c$ and the region obtained by joining them to the upper half-plane by $N$. Taking in $N$ the ordinary topology of the complex plane, we see at once from their expressions as integrals in the various cases that the lengths corresponding to $a_{1}, a_{2}$, and $a_{3}$ depend continuously on a point moving in this region.

No two points of $N$ could give the same double ratio $a_{1}: a_{2}: a_{3}$. Indeed, if they did, the corresponding canonical domains would have to be similar. Thus we should be led to a conformal map of the upper half $z$-plane on itself leaving invariant six boundary points and hence the identity. However the points of the upper half-plane determining the mapping would be interchanged and we should be led to a contradiction.

We shall now make a more detailed study of this correspondence. Since only the ratio $a_{1}: a_{2}: a_{3}$ is of importance we may take $a_{1}, a_{2}, a_{3}$ as trilinear coordinates of a point. Further, all three are non-negative so we shall be con- 
cerned with the interior of the triangle of reference. As we have observed in the discussion of the canonical domains, to the interior points of $H$ correspond points of the triangle for which $a_{1}, a_{2}$, and $a_{3}$ satisfy the triangle law $a_{1}+a_{2}>a_{3}, a_{2}+a_{3}>a_{1}, a_{3}+a_{1}>a_{2}$. At the boundary points of $H$ in the segments $z_{1} z_{2}, z_{3} z_{4}$, and $z_{5} z_{6}$ one of these becomes an equality. As we describe the segment $z_{1} z_{2}$ the image point describes a segment $Z_{1} Z_{2}$ on the line $a_{2}=a_{3}+a_{1}$. As we describe the segment $z_{2} z_{3}$ the image point passes on a certain continuous curve $Z_{2} Z_{3}$ over to the line $a_{3}=a_{1}+a_{2}$. In this way we see that to the boundary of $H$ corresponds the closed curve $Z_{1} Z_{2} Z_{3} Z_{4} Z_{5} Z_{6}$ where $Z_{i}$ corresponds to $z_{i}, i=1, \cdots, 6$. Of the curves $Z_{2} Z_{3}, Z_{4} Z_{5}, Z_{6} Z_{1}$ we can say that each is met at most once by any line through the opposite vertex of the triangle of reference. Clearly, by the uniqueness property of the canonical

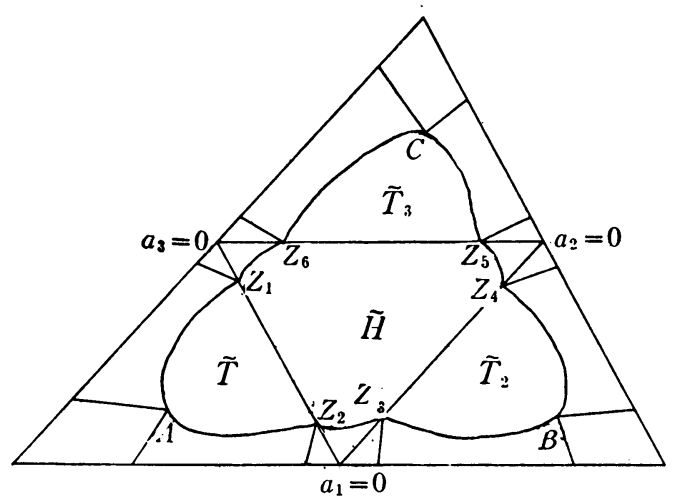

FIG. 4

domain corresponding to the double ratio $a_{1}: a_{2}: a_{3}$, the curve cannot cover any point twice. Suppose that, say, $Z_{4} Z_{5}$ had two distinct points of intersection with a line through the point $(0,1,0)$. The corresponding canonical domains would be of the second type. They would correspond to double ratios $a_{1}: a_{2}: a_{3}$ and $a_{1}: a_{2}^{\prime}: a_{3}, a_{2}>a_{2}^{\prime}$, where the second would refer to the point farther from the vertex. If we consider the problem $P\left(a_{1}, a_{2}^{\prime}, a_{3}\right)$ for the canonical domain corresponding to the first point the familiar argument shows us that $\rho \equiv 1$ would be a minimizing function. The above situation is now seen to be impossible by the same method as is used to prove the uniqueness property of canonical domains.

As we describe the segment $z_{1} a$ of the boundary of $T_{1}$, this is equivalent in the original $z$-plane to having $z^{* *}$ fixed at $z_{1}$ and letting $z^{*}$ run from $z_{1}$ to $z_{2}$. The image point in the triangle describes a continuous curve from $Z_{1}$ to a point $A$ at which the canonical domain becomes completely degenerate, that is, $\beta_{1}=0, \beta_{3}=0$. As we describe the segment $a z_{2}$, this is equivalent in the original $z$-plane to having $z^{*}$ fixed at $z_{2}$ and letting $z^{* *}$ run from $z_{1}$ to $z_{2}$. The image point describes a continuous curve from $A$ to $Z_{2}$. Similar remarks hold for the boundary segments of the triangles $T_{2}$ and $T_{3}$. As before, it can be 
shown for these curves that they are met at most once by any line through the opposite vertex.

Let $\tilde{N}$ be the region bounded by the curve $Z_{1} A Z_{2} Z_{3} B Z_{4} Z_{5} C Z_{6}$ with $\tilde{H}, \tilde{T}_{1}, \tilde{T}_{2}, \tilde{T}_{3}$ the parts of $\tilde{N}$ corresponding to $H, T_{1}, T_{2}, T_{3}$ in $N$. Let $F$ be a boundary point of $\tilde{N}$ and let us draw the line segment through it to the perimeter of the triangle whose continuation passes through the opposite vertex. For definiteness suppose $F$ lies on $A B$. Then for the points of this segment the ratio $a_{2}: a_{3}$ has the same value as for $F$ while $a_{1}: a_{2}$ (or $a_{1}: a_{3}$ ) is smaller. As in the discussion of the boundary curves of $\tilde{N}$ given above, the canonical domain corresponding to $F$ does for all these points. Similar remarks naturally hold for the sides $B C$ and $C A$. In the same manner we see that all points in the three quadrilaterals in the corners of the triangle obtained by the above construction correspond, respectively, to the three completely degenerate cases.

Thus a point in the triangle corresponding to a point of the region $N$ must lie in $\tilde{N}$, while to different points of $N$ correspond different points of $\tilde{N}$. Further, the boundaries of these regions are in $(1,1)$ continuous (and thus topological) correspondence. Then from the invariance of domain we deduce that the points of the two regions are in topological correspondence. This completes the proof that to any ratio $a_{1}: a_{2}: a_{3}$ we obtain a canonical domain of one of the prescribed types.

4. We shall now proceed to consider a mapping problem for the hexagon. Let $H^{\prime}$ be a second hexagon with vertices $1^{\prime}, 2^{\prime}, 3^{\prime}, 4^{\prime}, 5^{\prime}, 6^{\prime}$, the order around the boundary being the same as before. We shall denote all entities in the corresponding way as for $H$ with the addition of primes.

We desire to investigate when it is possible to map this second hexagon conformally into the first in such a way that the sides $1^{\prime} 2^{\prime}, 3^{\prime} 4^{\prime}, 5^{\prime} 6^{\prime}$ lie along the sides $12,34,56$.

We shall prove the following result:

TheOREM 2. A necessary and sufficient condition for the possibility of the mapping as above is that

$$
M\left(a_{1}, a_{2}, a_{3}\right) \geqq M^{\prime}\left(a_{1}, a_{2}, a_{3}\right)
$$

for all triples $a_{1}, a_{2}, a_{3}$.

The necessity of this condition is seen precisely as for the pentagon. Indeed, suppose we have the mapping performed as above. Then any curve of $C_{1}^{\prime}$ is identical with a curve of $C_{1}$, any curve of $C_{2}^{\prime}$ identical with a curve of $C_{2}$, and any curve of $C_{3}^{\prime}$ identical with a curve of $C_{3}$. Hence any admissible function $\rho$ for $P\left(a_{1}, a_{2}, a_{3}\right)$ gives an admissible function $\rho^{\prime}$ for $P^{\prime}\left(a_{1}, a_{2}, a_{3}\right)$ by assigning the same value at the same point (taken in the plane for $H$ ). Then

$$
\iint_{H} \rho^{2} d x d y \geqq \iint_{H^{\prime}} \rho^{\prime 2} d x d y
$$


Since there are possibly other admissible functions for $P^{\prime}\left(a_{1}, a_{2}, a_{3}\right)$ we have further

$$
\min _{\rho} \iint_{H} \rho^{2} d x d y \geqq \min _{\rho^{\prime}} \iint_{H^{\prime}} \rho^{\prime 2} d x d y \text {. }
$$

That is,

$$
M\left(a_{1}, a_{2}, a_{3}\right) \geqq M^{\prime}\left(a_{1}, a_{2}, a_{3}\right) .
$$

We now proceed to prove the sufficiency of the given condition. To every point of the domain $\tilde{N}$ we select a triple $a_{1}, a_{2}, a_{3}$. These values are to vary continuously with position in the triangle. Since no point of $\tilde{N}$ has any of its homogeneous coordinates zero we may take, for example, $a_{1}$ to have a constant value. Let $\beta_{1}, \beta_{2}, \beta_{3}$ be the quantities previously defined associated with the canonical domain corresponding to the point $\left(a_{1}, a_{2}, a_{3}\right)$. For the points of $\tilde{N}$ the results of $\$ 2$ relating the dimensions of the canonical domain and the quantities $a_{1}, a_{2}, a_{3}$ are all equalities. We shall now show that $\beta_{1}, \beta_{2}, \beta_{3}$ vary continuously with position in the triangle. Indeed for $\tilde{H}$ and its boundary we have the equations:

$$
\beta_{1}+\beta_{2}=A_{3} A_{4}, \quad \beta_{2}+\beta_{3}=A_{5} A_{6}, \quad \beta_{3}+\beta_{1}=A_{1} A_{2},
$$

while for $\widetilde{T}_{3}$, say, we have:

$$
\begin{gathered}
\beta_{1}+\beta_{2}=A_{3} A_{4}, \quad \beta_{2}+\beta_{3}=A_{5} A_{6}\left(=A_{5} \overline{\bar{A}}+\overline{\overline{A A}}+\bar{A} A_{6}\right)-\bar{A} \bar{A}, \\
\beta_{3}+\beta_{1}=A_{1} A_{2} .
\end{gathered}
$$

These lengths can be expressed in terms of integrals which vary continuously and, at the points where $\tilde{H}$ and $\widetilde{T}_{3}$ meet, the length $\bar{A} \bar{A}$ is zero. Similar remarks hold for $\tilde{T}_{1}$ and $\tilde{T}_{2}$. Finally, from what we have learned about canonical domains for points of the triangle outside of $\tilde{N}$, we see that $\beta_{1}, \beta_{2}, \beta_{3}$ will vary continuously with respect to position in the triangle. Naturally the same is true for $\beta_{1}^{\prime}, \beta_{2}^{\prime}, \beta_{3}^{\prime}$, the corresponding quantities for $H^{\prime}$.

Now we can express the condition

$$
M\left(a_{1}, a_{2}, a_{3}\right) \geqq M^{\prime}\left(a_{1}, a_{2}, a_{3}\right)
$$

as

$$
a_{1} \beta_{1}+a_{2} \beta_{2}+a_{3} \beta_{3} \geqq a_{1} \beta_{1}^{\prime}+a_{2} \beta_{2}^{\prime}+a_{3} \beta_{3}^{\prime}
$$

which can in turn be written

$$
a_{1}\left(\beta_{1}-\beta_{1}^{\prime}\right)+a_{2}\left(\beta_{2}-\beta_{2}^{\prime}\right)+a_{3}\left(\beta_{3}-\beta_{3}^{\prime}\right) \geqq 0 .
$$

This can be interpreted as saying that, taken in a Cartesian coordinate system, with axes $O z, O y, O z$ say, the vectors $\left(a_{1}, a_{2}, a_{3}\right)$ and $\left(\beta_{1}-\beta_{1}^{\prime}, \beta_{2}-\beta_{2}^{\prime}\right.$, $\beta_{3}-\beta_{3}^{\prime}$ ) form an angle not greater than a right angle (assuming the latter vector is not the null vector). Since the first vector must lie in the octant 
$(x+, y+, z+)$, the second cannot lie in the interior of the octant $(x-, y-, z-)$. As the triple $a_{1}, a_{2}, a_{3}$ varies, the end point of the vector $\left(\beta_{1}-\beta_{1}^{\prime}, \beta_{2}-\beta_{2}^{\prime}, \beta_{3}-\beta_{3}^{\prime}\right)$ varies continuously. We shall regard the image under this of the region $\tilde{N}$ defined as above. (This refers to $H$, not $H^{\prime}$.) As we have seen, for these points, the sides $A_{2} A_{3}, A_{4} A_{5}$, and $A_{6} A_{1}$ actually have the lengths $a_{1}, a_{2}$, and $a_{3}$.

The image point $A^{*}$ of $A$ will lie in the closed octant $(x-, y+, z-)$, the image point $B^{*}$ of $B$ in the closed octant $(x-, y-, z+)$, the image point
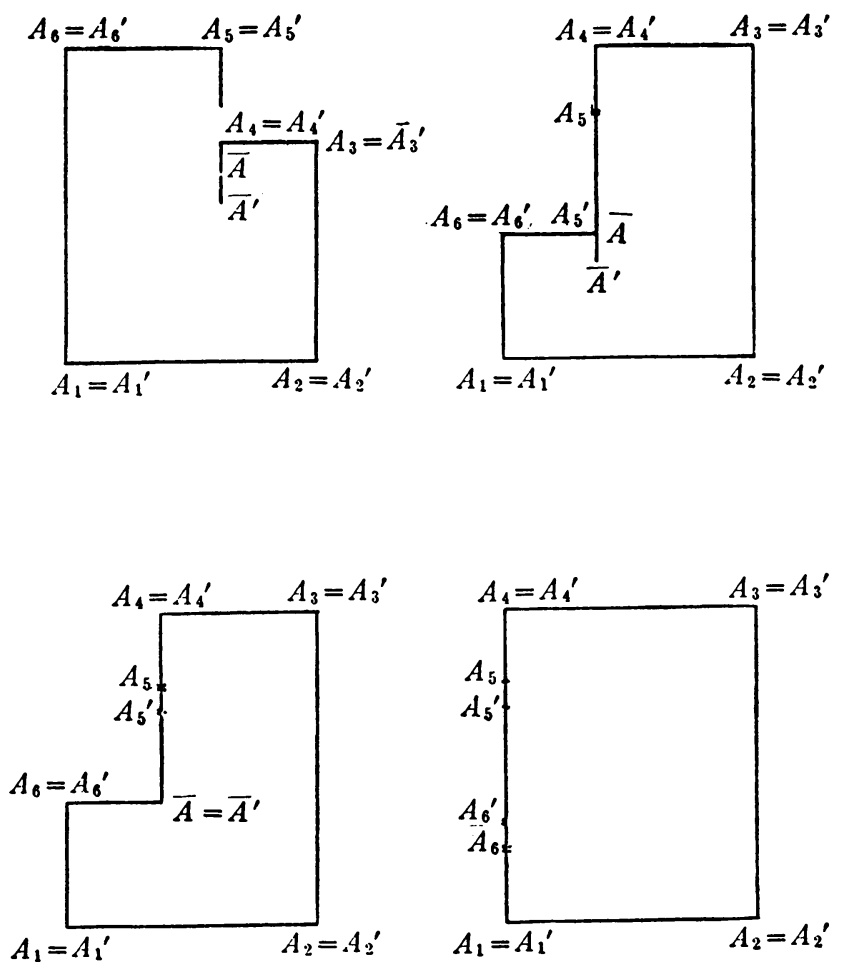

FIG. 5

$C^{*}$ of $C$ in the closed octant $(x+, y-, z-)$. The image of the side $A B$ will lie in the three closed octants $(x-, y+, z+),(x-, y+, z-),(x-, y-, z+)$. Similar remarks hold for the sides $B C$ and $A C$.

If the image surface passes through the origin we have for that value of the triple $a_{1}, a_{2}, a_{3} ; \beta_{1}=\beta_{1}^{\prime}, \beta_{2}=\beta_{2}^{\prime}$, and $\beta_{3}=\beta_{3}^{\prime}$. If $\beta_{1}, \beta_{2}, \beta_{3}$ are all positive the canonical domains are identical. If any of the $\beta_{i}$ vanishes so does the corresponding $\beta_{i}^{\prime}$ and the suitable side of the canonical domain for $H^{\prime}$ has length at least $a_{i}$. If the canonical domain for $H$ is of the second type, that for $H^{\prime}$ is also of the second type. If the canonical domain for $H$ is of the fourth type, that for $H^{\prime}$ is of the second or fourth type. If the canonical domain for $H$ is of the fifth type, so is that for $H^{\prime}$. In any case we can lay the canonical domain for $H^{\prime}$ into the canonical domain for $H$. To this corresponds the required 
mapping of $H^{\prime}$ into $H$. This is illustrated in Fig. 5 for the four typical degenerate cases.

The image surface, if it does not pass through the origin, can be projected continuously from that point onto a sphere with center there. The images of the vertices and sides of $A B C$ will fall on the parts of the sphere in the octants mentioned above. Let $P Q R$ be the equilateral right-angled
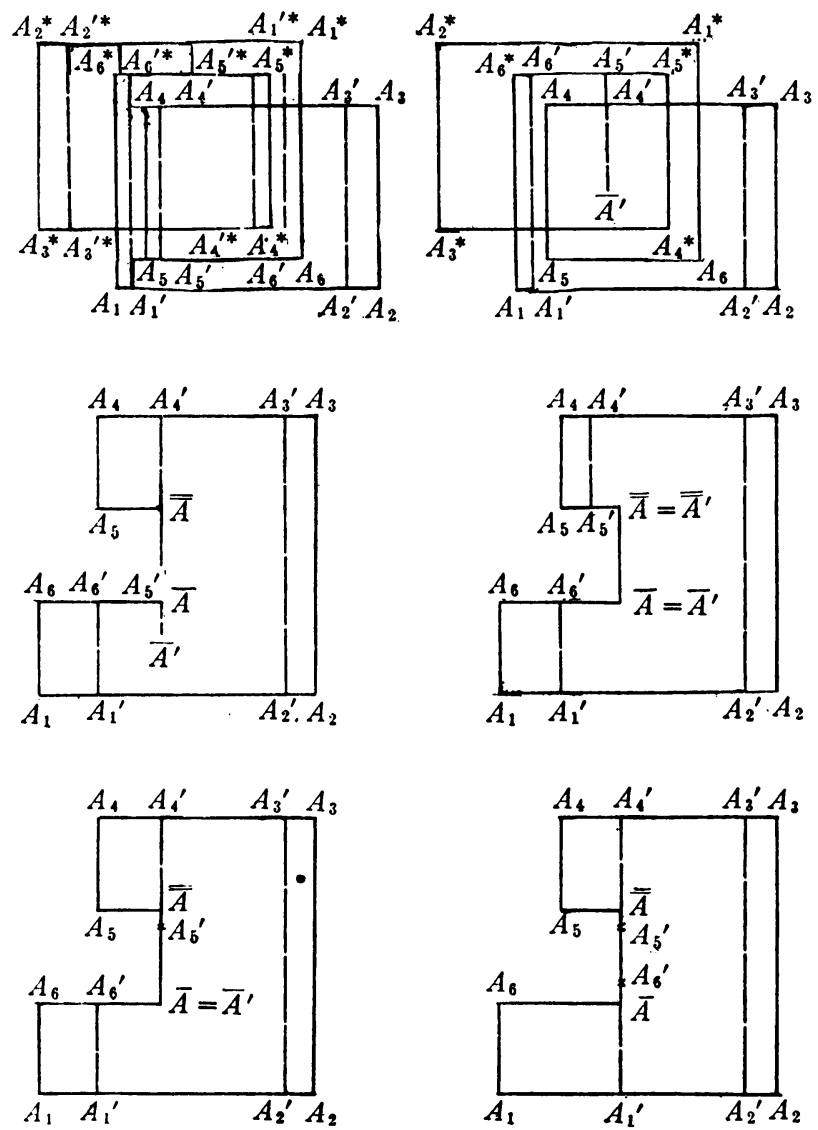

FIG. 6

triangle cut out on the surface of the sphere by the first octant, $P, Q, R$ being on the $x$-, $y$-, and $z$-axes respectively. Let $U, V, W$ be the mid-points of the sides $R P, P Q, Q R$ and $S$ the centroid of the triangle.

We shall now deform the image of $\tilde{N}$ on the sphere as follows: points of the triangle $P Q R$ will be left fixed, points exterior to $P Q R$ will be projected from $S^{\prime}$, the antipodal point of $S$ on the sphere, along great circles through $S^{\prime}$ and $S$, onto the perimeter of $P Q R$. Since the image does not cover $S^{\prime}$, this is a continuous mapping. The image of $A$ will lie on $Q V$ or $Q W$, that of $B$ on $R W$ or $R U$, that of $C$ on $P U$ or $P V$. The image of $A B$ will lie along $Q V, Q W, R W$, $R U$ while similar remarks hold for $B C$ and $C A$. 
The pencil of great circles through $S$ induces a correspondence between points of the perimeter of $P Q R$ and their opposite points on this perimeter. It is clear that the image of $A$ does not lie opposite to $Q$, the image of $B$ does not lie opposite to $R$, and the image of an interior point of $A B$ does not lie opposite to an interior point of $Q R$. Similar remarks hold for $B C$ and $C A$. Thus the mapping of the boundary of $\tilde{N}$ into $P Q R$ can be deformed in such a way that $A, B, C$ go into $Q, R, P, A B$ goes homeomorphically into $Q R, B C$ homeomorphically into $R P, C A$ homeomorphically into $P Q$. That is, provided we take suitable orientations, the perimeter of $\tilde{N}$ is mapped into the perimeter of $P Q R$ with degree 1 . Thus the previous mapping was also of degree 1 .

Finally we can project the triangle $P Q R$ of the sphere from the center $O$ onto the tangent plane at $S$. It will have as image a triangle and we shall obtain a continuous mapping of $\tilde{N}$ into this triangle such that the perimeter of $\tilde{N}$ is mapped into the perimeter of this last triangle (suitably oriented) with degree 1. Thus the order of the mapping of the perimeters about $S$ is 1 and the degree of the mapping of the areas over $S$ is 1 . Thus $S$ is covered by the image. Similarly every point of the triangle is covered.

This means that there was originally a vector $\left(\beta_{1}-\beta_{1}^{\prime}, \beta_{2}-\beta_{2}^{\prime}, \beta_{3}-\beta_{3}^{\prime}\right)$ lying in the octant $(x+, y+, z+)$. Thus there exists a triple $a_{1}, a_{2}, a_{3}$ with $\beta_{1}>\beta_{1}^{\prime}, \beta_{2}>\beta_{2}^{\prime}, \beta_{3}>\beta_{3}^{\prime}$. The corresponding canonical domain for $H$ will be of the first or third type. If it is of the first type the corresponding canonical domain for $H^{\prime}$ will be of the first or second type. If it is of the third type the corresponding canonical domain for $H^{\prime}$ will be of the second, third, fourth, or fifth type. In any case we can lay the canonical domain for $H^{\prime}$ into the canonical domain for $H$. This is illustrated in Fig. 6 for typical cases.

We observe that in the first case the canonical domain for $H^{\prime}$ lies symmetrically about the branch point, hence we get the required mapping of $H^{\prime}$ into $H$ by taking just one sheet. In the second case no two points of the canonical domain for $H^{\prime}$ lie symmetrically with respect to the branch point, hence we get the required mapping by projecting into one sheet. Hence in any case we obtain the required mapping of $H^{\prime}$ into $H$.

5. Further we observe that if we have the strict inequality

$$
M\left(a_{1}, a_{2}, a_{3}\right)>M^{\prime}\left(a_{1}, a_{2}, a_{3}\right)
$$

only the second possibility above can occur. Thus the interior of $H^{\prime}$ can be mapped into a proper subdomain of the interior of $H$. However, if we apply the same argument as for the pentagon we see that we can have equality without coincidence of the interiors when the canonical domain for $H^{\prime}$ is of the second type. (See Fig. 5.) For the nondegenerate cases, when a mapping is possible, equality means conformal equivalence.

We may now state the following complement to Theorem 2.

THEOREM 2a. $H$ and $H^{\prime}$ are conformally equivalent if and only if 


$$
M\left(a_{1}, a_{2}, a_{3}\right)=M^{\prime}\left(a_{1}, a_{2}, a_{3}\right)
$$

for all triples $a_{1}, a_{2}, a_{3}$.

$H^{\prime}$ can be mapped in the required manner into $H$ so that its interior goes into a proper subdomain of the interior of $H$ if

$$
M\left(a_{1}, a_{2}, a_{3}\right)>M^{\prime}\left(a_{1}, a_{2}, a_{3}\right)
$$

for all triples $a_{1}, a_{2}, a_{3}$.

\section{Chapter IV. The TRIPLy-CONNECTED DOMAIN}

1. We shall now enter into some considerations for a triply-connected domain, among them a mapping problem. We shall regard a triply-connected domain $D$, none of whose boundary continua $K_{1}, K_{2}, K_{3}$ reduces to a single point. Such a domain can be mapped on a domain bounded by three circles. There will be a circle orthogonal to these three and for convenience we can take this to be a straight line. This line divides the domain into two symmetric hexagons, $H$ and $\hat{H}$. With a view to discussing the conformal mapping of one such domain into another, we shall define a module for a triply-connected domain by means of the following extremal problem, $\mathrm{P}\left(a_{1}, a_{2}, a_{3}\right)$.

Problem $P\left(a_{1}, a_{2}, a_{3}\right)$ : Let $C_{1}$ denote the class of Jordan curves $\gamma_{1}$, lying in the domain $D$ and separating the contour $K_{1}$ from the contours $K_{2}$ and $K_{3}$. Let $C_{2}$ denote the similar class for $K_{2}$ and $C_{3}$ the similar class for $K_{3}$.

Let $\rho$ denote a non-negative function defined over $D$, of integrable square and such that the integrals $\oint_{\gamma_{1} \rho} \rho d z\left|, \oint_{\gamma_{2} \rho} \rho d z\right|, \oint_{\gamma_{3} \rho}|d z|$ exist (possibly having the value $+\infty$ ) with

$$
\oint_{\gamma_{1}} \rho|d z| \geqq 2 a_{1}, \quad \oint_{\gamma_{2}} \rho|d z| \geqq 2 a_{2}, \quad \oint_{\gamma_{3}} \rho|d z| \geqq 2 a_{3}
$$

where $a_{1}, a_{2}, a_{3}$ are certain three non-negative numbers. It is required to determine a $\rho$ such that

$$
\iint_{D} \rho^{2} d x d y \quad(z=x+i y)
$$

is a minimum. The corresponding minimum will be denoted by $\mathcal{X}\left(a_{1}, a_{2}, a_{3}\right)$ and called the module of the domain.

2. We shall proceed to prove the following.

TheOREM 3. $\mathcal{H}\left(a_{1}, a_{2}, a_{3}\right)$ exists and is equal to $2 M\left(a_{1}, a_{2}, a_{3}\right)$ where this latter is the module of $H$ (which is naturally also the module of $\widehat{H}$ ). We should remark that the vertices of $H$ must be numbered so that the curves of class $C_{i}$ for $H$ are portions of curves of class $C_{i}$ for $D$.

Indeed we note first that, given any function $\rho$.admissible for $P\left(a_{1}, a_{2}, a_{3}\right)$, 
extending its definition by symmetry to $\widehat{H}$ we obtain a function admissible for $P\left(a_{1}, a_{2}, a_{3}\right)$. Further

$$
\iint_{D} \rho^{2} d x d y=2 \iint_{H} \rho^{2} d x d y
$$

thus

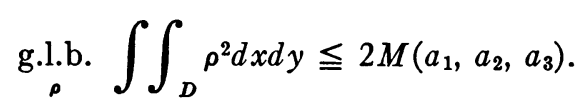

Again, given any function $\rho$ admissible for $P\left(a_{1}, a_{2}, a_{3}\right)$, let $\rho$ be the function obtained by reflection in the line of symmetry. Then

$$
\iint_{D} \rho^{2} d x d y=\iint_{D} \hat{\rho}^{2} d x d y .
$$

Moreover

$$
\begin{aligned}
\frac{1}{2} \iint_{D} \rho^{2} d x d y+\frac{1}{2} \iint_{D} \hat{\rho}^{2} d x d y & \\
& =\iint_{D}\left(\frac{\rho+\hat{\rho}}{2}\right)^{2} d x d y+\iint_{D}\left(\frac{\rho-\hat{\rho}}{2}\right)^{2} d x d y
\end{aligned}
$$

Finally $(\rho+\hat{\rho}) / 2$ is an admissible function in $P\left(a_{1}, a_{2}, a_{3}\right)$ so that

$$
\iint_{D}\left(\frac{\rho+\hat{\rho}}{2}\right)^{2} d x d y \geqq 2 M\left(a_{1}, a_{2}, a_{3}\right) \text {. }
$$

Thus

$$
\iint_{D} \rho^{2} d x d y \geqq 2 M\left(a_{1}, a_{2}, a_{3}\right)
$$

and

$$
\underset{\rho}{\text { g.l.b. }} \iint_{D} \rho^{2} d x d y \geqq 2 M\left(a_{1}, a_{2}, a_{3}\right) \text {. }
$$

This proves that

$$
\underset{\rho}{\text { g.l.b. }} \iint_{D} \rho^{2} d x d y=2 M\left(a_{1}, a_{2}, a_{3}\right) \text {. }
$$

The minimizing function for the hexagon gives at once the minimizing function for the triply-connected domain so that the minimum $\mathscr{N}\left(a_{1}, a_{2}, a_{3}\right)$ is actually attained. This proves the result.

We see that we have incidentally proved that the minimizing function 
takes almost everywhere the same value at points symmetric with respect to the line of symmetry. This could be seen in numerous other ways also.

3. We now treat a mapping problem for triply-connected domains. We shall denote by $D^{\prime}$ a triply-connected domain (none of whose boundary continua $K_{1}^{\prime}, K_{2}^{\prime}, K_{3}^{\prime}$ reduces to a single point) and by $\mathcal{H C}^{\prime}\left(a_{1}, a_{2}, a_{3}\right)$ its module.

THEOREM 4. A necessary and sufficient condition that the triply-connected domain $D^{\prime}$ can be mapped into the triply-connected domain $D$ in such a way that the contours have the same topological situation is that

$$
\mathcal{H}\left(a_{1}, a_{2}, a_{3}\right) \geqq \mathcal{H}^{\prime}\left(a_{1}, a_{2}, a_{3}\right)
$$

for all triples $a_{1}, a_{2}, a_{3}$.

Indeed, let us suppose that such a mapping is possible. Then any curve of $C_{1}^{\prime}$ goes into a curve of $C_{1}$, any curve of $C_{2}^{\prime}$ into a curve of $C_{2}$, and any curve of $C_{3}^{\prime}$ into a curve of $C_{3}$. Hence any admissible function $\rho$ for $\mathcal{P}\left(a_{1}, a_{2}, a_{3}\right)$ gives an admissible function $\rho^{\prime}$ for $\mathcal{P}^{\prime}\left(a_{1}, a_{2}, a_{3}\right)$ by assigning the same value at the same point. Then

$$
\iint_{D} \rho^{2} d x d y \geqq \iint_{D^{\prime}} \rho^{\prime 2} d x d y
$$

There are possibly other admissible functions for $\mathcal{P}^{\prime}\left(a_{1}, a_{2}, a_{3}\right)$ so that

$$
\mathscr{H}\left(a_{1}, a_{2}, a_{3}\right) \geqq \mathcal{H}^{\prime}\left(a_{1}, a_{2}, a_{3}\right) .
$$

This demonstrates the necessity of the condition.

To show the sufficiency of the condition we note that it implies

$$
M\left(a_{1}, a_{2}, a_{3}\right) \geqq M^{\prime}\left(a_{1}, a_{2}, a_{3}\right)
$$

for the modules of the corresponding hexagons. Thus $H^{\prime}$ can be mapped into $H$ as in the mapping problem for the hexagon. Extending this by symmetry to a mapping of $\widehat{H}^{\prime}$ into $\widehat{H}$ we obtain the required mapping of $D^{\prime}$ into $D$.

From this we deduce at once the following result.

THEOREM 5. If the triply-connected domain $D^{\prime}$ can be mapped into the triplyconnected domain $D$ in such a way that corresponding contours have the same topological situation, another such mapping can be performed so that the lines of symmetry coincide.

4. We shall now give a brief discussion of the possibility of equality in Theorem 4. By Theorem 5 it is enough to regard the mapping of the corresponding hexagons. Thus if we always have equality the domains are conformally equivalent. If we always have inequality we have the interior of one mapped into a proper subdomain of the interior of the other. However the converse is not true as can be seen by regarding the cases for the hexagon where we can have both equality and inequality. We can obtain a counter- 
example by taking a domain $D$ bounded by three circles and making a slit along the line of symmetry, either out from one contour or out from two contours facing each other on a segment of the line of symmetry interior to the domain. It is conceivable that the corresponding $D^{\prime}$ would admit also an asymmetric mapping into $D$. It is rather hard to say how we might characterize such a domain.

Actually it is not too difficult to see that those subdomains of $D$ for which equality can occur in some case admit no other mapping into $D$.

5. Finally we can readily see that there exist triply-connected domains neither of which can be mapped into the other for any numbering of the contours. It is again enough to work with hexagons. Let us take two canonical domains of the first type. (It is readily seen that they actually are canonical domains for suitable hexagons.) Let them both have the same $a_{1}, a_{2}, a_{3}$, all equal, and the same area. Let one have all its $\beta$ 's equal, the other have them all different from this common value. If one could be mapped into the other, since we have a nondegenerate case, equality of the modules would mean conformal equivalence, which is out of the question.

\section{BIBLIOGRAPHY}

1. L. Ahlfors, Untersuchungen zur Theorie der konformen Abbildung und der ganzen Funktionen, Acta Societatis Scientiarum Fennicae, N. S. A. vol. 1 (1930).

2. L. Ahlfors and A. Beurling, Invariants conformes et problèmes extrémaux, Dixième Congrès des Mathématiciens Scandinaves, 1946.

3. A. Beurling, Études sur un problème de majoration, Thesis, 1933.

4. H. Grötzsch, Über einige Extremalprobleme der konformen Abbildung, Berichte über die Verhandlungen der Sächsischen Akademie der Wissenschaften zu Leipzig, Math-Phys. Klasse vol. 80 (1928) pp. 366-376.

5. O. Teichmüller, Extremale quasikonforme Abbildungen und quadratische Differentiale, Abhandlungen der Preussischen Akademie der Wissenschaften, Math-naturw. Klasse, No. 22, 1939.

Harvard UNIVERSITY, Cambridge, Mass. 